\title{
Cavitation instabilities between fibres in a metal matrix composite
}

\section{Tvergaard, Viggo}

\section{Published in:}

Acta Mechanica

Link to article, DOI:

10.1007/s00707-015-1511-3

Publication date:

2016

Document Version

Peer reviewed version

Link back to DTU Orbit

Citation (APA):

Tvergaard, V. (2016). Cavitation instabilities between fibres in a metal matrix composite. Acta Mechanica, 227(4), 993-1003. https://doi.org/10.1007/s00707-015-1511-3

\section{General rights}

Copyright and moral rights for the publications made accessible in the public portal are retained by the authors and/or other copyright owners and it is a condition of accessing publications that users recognise and abide by the legal requirements associated with these rights.

- Users may download and print one copy of any publication from the public portal for the purpose of private study or research.

- You may not further distribute the material or use it for any profit-making activity or commercial gain

- You may freely distribute the URL identifying the publication in the public portal

If you believe that this document breaches copyright please contact us providing details, and we will remove access to the work immediately and investigate your claim. 


\title{
CAVITATION INSTABILITIES BETWEEN FIBRES IN A METAL MATRIX COMPOSITE
}

\author{
Viggo Tvergaard \\ Department of Mechanical Engineering, Solid Mechanics \\ Technical University of Denmark, DK-2800 Kgs. Lyngby, Denmark
}

\begin{abstract}
Short fibre reinforced metal matrix composites (MMC) are studied here to investigate the possibility that a cavitation instability can develop in the metal matrix. The high stress levels needed for a cavitation instability may occur in metal ceramic systems due to the constraint on plastic flow induced by bonding to the ceramics that only show elastic deformation. In a MMC the stress state in the metal matrix is highly non-uniform, varying between regions where shear stresses are dominant and regions where hydrostatic tension is strong. An Al-SiC whisker composite with a periodic pattern of transversely staggered fibres is here modelled by using an axisymmetric cell model analysis. First the critical stress level is determined for a cavitation instability in an infinite solid made of the $\mathrm{Al}$ matrix material. By studying composites with different distributions and aspect ratios of the fibres it is shown that regions between fibre ends may develop hydrostatic tensile stresses high enough to exceed the critical level for a cavitation instability. For cases where a void is located in such regions it is shown that unstable cavity growth develops when the void is initially much smaller than the highly stressed region of material.
\end{abstract}

Keywords: Void growth; Plasticity; Cavitation instability; Micromechanics; Metal Matrix Composite.

\section{INTRODUCTION}

Metal matrix composites (MMC) are an important type of the metal-ceramic systems where the presence of elastic fibres gives a constraint on plastic flow, which tends to increase the macroscopic stress level in the material. In a short fibre reinforced material this will also increase the stress level in the metal matrix so that a cavitation instabilitiy may occur.

Reinforcement of metals by short brittle fibres increases the stiffness and tensile strength, but also results in poor ductility and low fracture toughness due to debonding of the fibre-matrix interface and fibre breakage (McDanels [1], Zok et al. [2], Mummery and Derby [3]). The variation in uniaxial tensile stress-strain behavior in response to variations of the matrix microstructure for a 2124 Al-SiC whisker composite has been investigated by Christman et al. [4]. In whisker composites it is often found that the fibres are rather well aligned as a result of the processing using either contracting flow or expanding flow in the extrusion (German and Bose [5]).

Micromechanical studies for metal matrix composites, by numerical cell model analyses, are an important tool for obtaining a parametric understanding of various material parameters. Such analyses allow for an accurate representation of the fibre shape and the material 
behavior in both fibres and matrix, thus leading to realistic stress and strain fields around the fibres (e.g. see Needleman et al. [6]). Axisymmetric cell models containing a single fibre have been used to study failure by debonding at the fibre-matrix interface and subsequent fibre pull-out (Nutt and Needleman [7], Tvergaard [8]) and have also been extended to consider both fibre breakage and debonding (Tvergaard [9,10,11]). The actual three-dimensional fibre distribution is only approximately represented in the axisymmetric analyses, but full 3D numerical cell model analyses (Levy and Papazian [12], Hom [13]) have confirmed a rather good agreement with axisymmetric results.

For a single small void in an infinite elastic-plastic solid under pure hydrostatic tension a critical stress level has been found, at which the void grows without bound (Bishop, Hill and Mott [14], Hill [15]). This unstable void expansion is driven by the elastic energy stored in the surrounding material. Such cavitation instabilities also occur under axisymmetric stress states (Huang et al. [16], Tvergaard et al. [17]), when the stress triaxiality is sufficiently high. Niordson and Tvergaard [18] have studied size-effects on the phenomenon and Legarth and Tvergaard [19] have analyzed void growth instabilities in a 3D stress state with anisotropic plasticity, and dynamic effects on cavitation instabilities have been studied by Kennedy et al. [20]. Also, in the context of nonlinear elasticity there has been much interest in cavitation instabilities (Ball [21], Horgan and Abeyarathne [22], Horgan and Polignone [23]).

In metal-ceramic systems the small strains in the ceramics give a constraint on plastic flow in the metal phase, which makes it more likely to reach high stress triaxialities in the metal. This was seen in experiments of Ashby, Blunt and Bannister [24] for a metal wire bridging a crack in a glass matrix, where fracture occurred by the growth of a single void to a diameter, which approached half the diameter of the metal wire. Also cavities growing in a thin metal layer used to bond two ceramics (Tvergaard [25]) tend to reach unstable growth due to the constraint on plasticity, if the voids are sufficiently small relative to the layer thickness.

Also in a metal matrix composite there is a constraint on plastic flow due to the presence of the fibres, which means that locally in the metal matrix between fibres the hydrostatic tension can be significantly larger than the overall hydrostatic tension applied to the composite. In the present paper a void embedded in the matrix between fibres is considered, and the dependence of the growth rate on the void radius, the fibre dimensions and fibre spacings are analysed. 


\section{PROBLEM FORMULATION}

The metal matrix composite (MMC) considered here is taken to have transversely staggered short fibres arranged in the periodic pattern illustrated in Fig. 1. As has been shown in previous studies [8,9] such transversely staggered patterns of brittle fibres give rise to high normal stresses on the fibre ends, but also result in large shear deformations in the metal matrix between fibre sides, when the composite is loaded in tension along the fibre direction. The transversely staggered array of fibres is chosen here, rather than transversely aligned fibres, because the staggered array shows a mixture of high shear stresses in parts of the metal between fibres and high normal stresses in other parts, as will often be the case in short fibre reinforced metals (e.g. see comparisons in [10]).

For tensile loading along the fibres combined with equal transverse loading in the directions perpendicular to the fibres a good estimate of the stresses and strains in this MMC is obtained by representing the material in terms of an axisymmetric unit cell model, as indicated by the hatched area in Fig. 1, and shown in more detail in Fig. 2. In some of the present analyses voids with initial radius $r_{v}$ are taken to be present in the material, as indicated in Fig. 2, while in other analyses there are no voids, as in Fig. 1. The cross-section perpendicular to the fibres (Fig. 1b) shows a square array of fibres with spacing $2 a_{c}$, and the

initial radius $r_{c}=(2 / \sqrt{\pi}) a_{c}$ of the axisymmetric model problem is chosen such that the fibre volume fraction of the cell is equal to that of the material illustrated in Fig. 1. With the initial cell length $\ell_{c}$, and the fibre geometry specified by the initial half length $\ell_{f}$ and radius $r_{f}$, the fibre volume fraction is $f=\left(r_{f}^{2} \ell_{f}\right) /\left(r_{c}^{2} \ell_{c}\right)$.

On the curved side of this circular cylindrical cell equilibrium and compatibility with the neighbouring cells has to be represented in an approximate manner, as described in $[8,9]$. A neighbouring cell is identical to that analyzed, but is rotated $180^{\circ}$ so that it points in the opposite direction (see Fig. 1a). Compatibility and equilibrium in the axial direction are directly specified in terms of the axial edge displacements and nominal tractions

$$
\begin{array}{ccc}
u^{1}=-U_{1} / 2, & T^{2}=0 & \text { at } x^{1}=0 \\
u^{1}=U_{1} / 2, & T^{2}=0 & \text { at } x^{1}=l_{c}
\end{array}
$$


where the constant $U_{1}$ is the increase of length of the cell, while the displacement components and the nominal traction components on reference base vectors are denoted by $u^{i}$ and $T^{i}$. In the radial direction compatibility is represented by the requirement that the total cross-sectional area (consisting of an equal number of cross-sections of the two types of neighbouring cells considered) is independent of the axial coordinate

$$
\begin{gathered}
u^{1}(\xi)=-u^{1}(\eta), \quad \text { for } \xi=\eta, \quad x^{2}=r_{c} \\
\left\{r_{c}+u^{2}(\xi)\right\}^{2}+\left\{r_{c}+u^{2}(\eta)\right\}^{2}=2\left\{r_{c}+U_{2}\right\}^{2}, \quad \text { for } \xi=\eta, x^{2}=r_{c}
\end{gathered}
$$

where $\xi$ and $\eta$ are distances from the bottom and the top of the cell, respectively, and $U_{2}$ is the radius increase at the centre of the cell (at $x^{1}=\ell_{c} / 2$ ). The equilibrium conditions on the cell side are specified as

$$
T^{1}(\xi)=T^{1}(\eta), \quad T^{2}(\xi)=T^{2}(\eta), \quad \text { for } \xi=\eta, x^{2}=r_{c}
$$

As $U_{1}$ denotes the increase of the length of the cell, and $U_{2}$ is the radius increase at the centre, the average logarithmic strains in the axial and transverse directions are $\varepsilon_{1}=\ln \left(1+U_{1} / \ell_{c}\right)$ and $\varepsilon_{2}=\ln \left(1+U_{2} / r_{c}\right)$, respectively. The average nominal stresses $\sum_{i j}$ are computed as the appropriate area averages of the microscopic nominal stress components on the surface (considering both the cell analysed and one of the neighbouring cells of opposite kind). The axial and transverse Cartesian stress components are $\sum_{11}$ and $\sum_{22}=\sum_{33}$ , respectively, for the axisymmetric problem, and all shear components vanish. Using the average strains $\varepsilon_{1}$ and $\varepsilon_{2}$, the average true stresses $\sigma_{1}$ and $\sigma_{2}$ are calculated from the nominal stress values.

The matrix material is taken to be described by $J_{2}$ flow theory with isotropic hardening. A convected coordinate, Lagrangian formulation of the field equations is used, in which $g_{i j}$ and $G_{i j}$ are metric tensors in the reference configuration and the current configuration, respectively, with determinants $g$ and $G$, and $\eta_{i j}=1 / 2\left(G_{i j}-g_{i j}\right)$ is the Lagrangian strain tensor. The contravariant components $\tau^{i j}$ of the Kirchhoff stress tensor on the current base vectors are related to the components of the Cauchy stress tensor $\sigma^{i j}$ by $\tau^{i j}=\sqrt{G / g} \sigma^{i j}$. The finite strain generalization of $J_{2}$ flow theory [26], gives an incremental 
stress-strain relationship of the form $\dot{\tau}^{i j}=L^{i j k \ell} \dot{\eta}_{k \ell}$, where $L^{i j k \ell}$ is the tensor of instantaneous moduli. The effective Mises stress is $\sigma_{e}=\left(3 s_{i j} s^{i j} / 2\right)^{1 / 2}$, with the stress deviator $s^{i j}=\tau^{i j}-G^{i j} \tau_{k}^{k} / 3$. The uniaxial stress-strain behaviour is represented by

$$
\varepsilon=\left\{\begin{array}{ccc}
\frac{\sigma}{E} & \text { for } & \sigma \leq \sigma_{y} \\
\frac{\sigma_{y}}{E}\left(\frac{\sigma}{\sigma_{y}}\right)^{n} & \text { for } & \sigma>\sigma_{y}
\end{array}\right.
$$

where $E$ is Young's modulus, $\sigma_{y}$ is the uniaxial yield stress, and $n$ is the strain hardening exponent.

The material properties for the MMC are chosen to be those measured in [4] for a whisker reinforced $2124 \mathrm{Al}-\mathrm{SiC}$ composite. Thus, the $\mathrm{Al}$ matrix is approximated by taking $\sigma_{Y} / E=0.005$ and $n=7.66$ in Eq. (1), with Poissons ratio $v=0.3$. For the brittle fibers the elastic properties are taken to be $E_{f}=5.7 E$ and $v_{f}=0.21$. The same material properties have been used in previous studies [8,9]. In [4] the fibre volume fraction was found to be around 0.13 , but here the fibre geometry is varied relative to the cell size to see the effect on growth of a void located as shown in Fig.2.

The void growth is the only type of damage considered here, while earlier studies have accounted for the possibility of fibre-matrix debonding as well as fibre breakage. The void considered here is taken to be located between fibre ends, which will tend to give high hydrostatic tension near the void. On the other hand, in the staggered array of fibres part of the load on a fibre will be carried by the neighbouring fibres through shear stresses in the metal matrix between the fibres. Therefore, the hydrostatic tension that drives the void growth will depend on geometry parameters such as the spacing between fibre ends, the transverse spacing between fibres, and the fibre aspect ratio.

In the numerical analysis for the axisymmetric unit cell, the displacement components on the base vectors of the cylindrical reference coordinate system are denoted $u^{i}$, and the Lagrangian strain tensor is given by

$$
\eta_{i j}=\frac{1}{2}\left(u_{i, j}+u_{j, i}+u_{, i}^{k} u_{k, j}\right)
$$


Here ()$_{, i}$ denotes the covariant derivative in the reference frame.

Numerical solutions are obtained by a linear incremental solution procedure, by expanding the principle of virtual work about the current state. The incremental equation is, to lowest order, equal to

$$
\int_{V}\left\{\Delta \tau^{i j} \delta \eta_{i j}+\tau^{i j} \Delta u_{, i}^{k} \delta u_{k, j}\right\} d V=\int_{A} \Delta T^{i} \delta u_{i} d A-\left[\int_{V} \tau^{i j} \delta \eta_{i j} d V-\int_{A} T^{i} \delta u_{i} d A\right]
$$

where $V$ and $A$ are, respectively, the volume and surface of the body in the reference configuration, $\Delta \tau^{i j}$ and $\Delta \eta_{i j}$ are the stress and strain increments, $T^{i}$ are contravariant components of the nominal surface tractions, etc. The bracketed terms are equilibrium corrections. The displacement fields are approximated in terms of 8-noded isoparametric elements, and volume integrals in the principle of virtual work are calculated by using 2x2 point Gauss integration within each element. An example of the mesh used for the computations is shown in Fig. 3. Here, inside the fibre volume the elastic fibre properties are used for the instantaneous moduli $L^{i j k \ell}$, while outside the elastic-plastic properties of the aluminium matrix are used. A special Rayleigh-Ritz finite element method [27] is used to implement the boundary conditions, to control the numerical stability during rapid void growth, and to enforce a fixed ratio, $\rho=\sigma_{2} / \sigma_{1}$, of the average true stresses in the transverse and axial directions, respectively.

\section{RESULTS}

First the critical stress level for the occurrence of cavitation instabilities is determined, for the particular metal used as matrix material here. Subsequently, a number of composites without voids are analyzed under different external loading to check whether or not the critical stress level is reached between fibres. Finally, the rate of void growth is analyzed for the same examples of short fibre reinforced metals.

\subsection{Cavitation instability limit in an infinite solid.}

Cavitation instability limits have been determined in a number of previous investigations $[16,17]$ for various power hardening solids, as a function of the remote stress state. These analyses were carried out by considering a single spherical void in an infinite solid subject to an axisymmetric remote stress state. The cavitation instability state has been reached when the 
void keeps growing for a fixed stress and strain state at infinity, so that the void growth is entirely driven by the stored elastic energy in the solid. In the present study the infinite solid is approximated by considering a circular cylindrical body with a central void so small that the void volume fraction is $10^{-10}$. As indicated by the insert in Fig. 4 the true stress in axial direction is $S$ and the transverse true stress is $T$. At the two dotted curves in in Fig. 4 plasticity occurs at infinity (see also [28]). It is seen that in the whole range of the stress ratio $T / S$ where plasticity is confined to a region around the central void cavitation occurs at a value $\sigma_{m} / \sigma_{Y}$ in the vicinity of 5.0 , where $\sigma_{m}$ is the mean value of the remote true stress.

\subsection{Stress states between fibres in an MMC with no voids.}

In these analyses for metal matrix composites like those illustrated in Figs. 1 to 3 there are no voids, but the local stress state is calculated at origo $\left(x_{1}=x_{2}=0\right)$, at the location where voids are shown in Figs. 2 and 3. In these calculations with no voids a uniform mesh is used in the region near origo, rather than a mesh focused on a void as shown in Fig. 3.

Fig. 5 shows the evolution of $\sigma_{m} / \sigma_{Y}$ vs. the average axial logarithmic strain $\varepsilon_{1}$ for four different composites subjected to an overall stress state characterized by the ratio $\rho=\sigma_{2} / \sigma_{1}=0.5$ of the average stresses on the composite. In the reference case the geometry of the unit cell and of the fibre are specified by $l_{c} / r_{c}=6, r_{f} / r_{c}=0.6$ and $l_{v} / r_{c}=0.8$. One of these three parameters is varied for each of the three other curves, as indicated by the parameter values for each curve. If the axial and transverse principal true stresses at origo are denoted by $S$ and $T$, thus referring to the stresses on the infinite solid in Fig. 4, the stress ratio $T / S$ is found to be initially rather low, but quickly grows to values between 0.8 and 0.85 , i.e. in the range of the cavitation curve shown in Fig. 4. Then, thinking about a void extremely much smaller than the metal region around origo, this void would behave much like a void in an infinite solid (Fig. 4), and since all four curves reach values of $\sigma_{m} / \sigma_{Y}$ well above 5.0 , it must be expected that a cavitation instability would be reached in all four cases. In fact three of the curves in Fig. 5 reach values of $\sigma_{m} / \sigma_{Y}$ as high as 10 or even 15, so in these cases a cavitation instability would be expected early on. It is seen in Fig. 5 that the values of the mean stress reach a maximum and then starts to decay, as would be expected since the plastic deformations change the aspect ratio of the metal region around origo, and the curves in Fig. 5 show that the stress level is quite sensitive to the initial value of this aspect ratio. 
In Fig. 6 curves are shown for the same four materials, now subjected to uniaxial tension $\rho=0.0$. Here the reference material does not reach the critical value of the mean stress near origo. High enough stresses are reached for $r_{f} / r_{c}=0.8$, where the larger fibre radius and thus smaller fibre spacing increase the mean stress, and for $l_{v} / r_{c}=0.4$, where the reduced spacing between fibre ends gives higher mean stress. These two cases also gave high mean stresses in Fig. 5. However, for $l_{c} / r_{c}=12$, where the cell aspect ratio is doubled and thus the fibre aspect ratio is more than doubled, the normalized mean stress in Fig. 6 just reaches a level around 5.0 , whereas in Fig. 5 this value exceeded 15. In Fig. 7 for the intermediate level $\rho=0.25$ of the average stress ratio three of the curves show values of the normalized mean stress well above the critical level for cavitation instabilities, whereas the reference case remains below that level.

\subsection{Rate of growth of a void located between fibre ends.}

The analyses here consider MMC's with a void located at origo, as illustrated in Figs. 2 and 3. The average stress ratio is $\rho=0.5$ as also considered in Fig. 5, and the material geometry is that of the reference material. It is known from previous studies of cavity growth in a finite metal volume [25] that a sufficiently small void relative to the metal volume shows a cavitation instability if the critical stress level is reached, whereas a larger void does not. According to Fig. 5 the reference material does reach the critical stress level at origo. For $r_{v} / r_{c}=0.001$ it is clear that the curve in Fig. 8 becomes vertical, so here the void is small enough to interact with the neighbouring metal region as if this was an infinite solid. Also for $r_{v} / r_{c}=0.01$ the curve is nearly vertical, very close to that for the ten times smaller initial void radius. However, for the two larger voids it is clear that there is no cavitation instability and that void growth requires further macroscopic straining of the metal matrix composite.

Fig. 9 shows deformed meshes corresponding to two of the curves in Fig. 8, both referring to the reference material. For $r_{v} / r_{c}=0.01$ Fig. 2a shows the deformed mesh at the stage where the normalized void volume has reached 4108, i.e. far above the top of the curve shown in Fig. 8. The deformed mesh in Fig. 9b corresponds to $r_{v} / r_{c}=0.1$ at a stage where the normalized void volume is 886, i.e. about halfway up on the curve in Fig. 8. This is the case used in Fig. 4 to illustrate an initial mesh. It is clear in Fig. 9b that the void has grown so close to the neighbouring fibres that the interaction with the surrounding metal cannot be similar to interaction with an infinite solid. It is noticed in both deformed meshes that there is 
no visible deformation of the fibres, which undergo only elastic deformations. It is also seen in Fig. 9b that expansion outside the average cell radius in one end of the unit cell gives less radial expansion at the other end of the unit cell, as is necessary due to the boundary conditions (3)-(4) on the cylindrical side of the unit cell, which represent interaction with a neighbouring unit cell identical to that analyzed, but rotated $180^{\circ}$ so that it points in the opposite direction (Fig. 1a).

Fig. 10 shows the evolution of the normalized void growth vs. axial strain for $\rho=0.5$ and $r_{v} / r_{c}=0.001$. The four different composite geometries are the same as those considered in Fig. 5 in the absence of any void. Based on the mean stress evolutions in Fig. 5 it was already expected that a cavitation instability would occur in all four cases provided that the void is sufficiently small, and this has already been seen in Fig. 8 for the reference case. The difference to the other three cases in Fig. 10 is that here the cavitation instability develops at smaller strains than that found for the reference case, as would also be expected based on the more rapid development of high mean stresses in Fig. 5.

A computation was also carried out for the same cell aspect ratio $l_{c} / r_{c}=6$, with $\rho=0.5$ and $r_{v} / r_{c}=0.001$, but with no fibres so that the volume occupied by fibres in Figs, 1,2 and 3 is here given the material properties of the $\mathrm{Al}$ matrix. This corresponds to all four curves in Fig. 10, but without fibres. Even though this curve was plotted into Fig. 10, it is not visible, since at $\varepsilon_{1}=0.1$ the normalized void volume $V / V_{0}$ has only grown to the value 1.99 from the initial value 1.0 . Thus, the constraint on plastic flow due to the fibres has a strong effect on the void growth behavior.

When the composites in Fig. 11 are subjected to uniaxial tension, $\rho=0$, rather than the higher transverse stress applied in Fig. 10, this results in much lower stress triaxiality in the vicinity of the void, as was also shown in Fig. 6. Based on Fig. 6 it is not surprising that the reference case shows rather slow void growth with no tendency to a cavitation instability, while the other three cases reach an instability at significantly higher strain values than those found in Fig. 10. It is noted that even though the macroscopic stress state has the low triaxiality $1 / 3$ corresponding to uniaxial tension, the constraint on plastic flow is high enough for some of the fibre arrangements to reach a cavitation instability locally in the metal between fibres.

Fig. 12 illustrates the intermediate stress state, $\rho=0.25$, between those in Figs. 10 and 11. The high levels of normalized void volume are here reached at larger axial strains than 
found in Fig. 10 and at smaller axial strains than found in Fig. 11. Also, the reference case does not quite reach a cavitation instability, as would be expected based on the mean stress level reached in Fig. 7 when there was no void present.

\section{DISCUSSION}

Cavitation instabilities in metals require a mean tensile stress many times the initial yield stress, as is known to occur in thin metal layers used to bond two ceramic bodies [24] or in metal particles bridging cracks in ceramics to add ductility [24]. The present studies show that also in a metal matrix composite, where the stress state in the metal is highly non-uniform, the constraint on plastic flow can be sufficient to result in unstable cavity growth in some points of the matrix.

For the particular aluminium alloy considered here it has been found that a cavitation instability can occur when the mean stress normalized by the initial yield stress is around 5 . It is known from previous studies [16,17] that this level varies with the material parameters, such that a more high hardening material, with a smaller value of $n$ in (6), results in an increased level of the critical stress for cavitation, while a higher value of the initial yield strain, $\sigma_{Y} / E$, gives a reduced level of the critical stress.

The metal matrix in an MMC does not everywhere reach very high tensile mean stresses. Thus, in the matrix between two parallel fibres pointing in the main tensile direction shear stresses dominate. But the focus here is on locations between ends of fibres, where a high mean stress tends to develop. It is shown that the mean stress level depends much on the ratio of the distance between fibre ends to fibre radius, on the fibre aspect ratio, and on the distance to parallel neighbour fibres. Comparison of the stress evolution at such locations between fibre ends shows that the fibres can give a constraint on plastic flow that results in stress levels even far higher than the cavitation limit.

The critical stress for a cavitation instability under a given remote stress state applies to the case of a single void in an infinite solid. If the solid is not infinite, it has to be so large relative to the void that there is enough stored elastic energy to drive the continued expansion of the void once the critical stress level has been reached. This effect is also found here (Fig. 8), where the initial void radii of 0.1 or 0.03 times the cell radius are not sufficiently small to allow for unstable cavity growth, but the unstable growth does develop for the smallest voids considered. Then, studying only small voids, with an initial radius of 0.001 times the 
cell radius, it was found here that unstable void growth does develop in the cases of fibre configurations and macroscopic stress states where analyses without voids have shown that the critical stress level would be exceeded.

In the cases where unstable cavity growth has been found (Figs. 8 to 12) this growth has been followed to normalized void volumes, $V / V_{0}$, exceeding 1600 , but this will not continue when the current void radius approaches the cell radius. In a previous study of void growth in a thin metal layer between ceramics [25] it has been possible, using remeshing, to follow unstable void growth up to a normalized void volune as high as $10^{9}$, but then neighbour voids have grown so large relative to their spacing that their growth develops into a standard coalescence mode. 


\section{REFERENCES}

1. D.L. McDanels, Analysis of stress-strain, fracture, and ductility behavior of aluminum matrix composites containing discontinuous silicon carbide reinforcement. Metall. Trans. A 16 (1985) 1105-1115.

2. F. Zok, J.D. Embury, M.F. Ashby, O. Richmond, The influence of pressure on damage evolution and fracture in metal-matrix composites. In: S.I. Andersen et al. (eds.), Mechanical and Physical Behaviour of Metallic and Ceramic Composites, Risø National Laboratory, Denmark, (1988) 517-526.

3. P. Mummery, B. Derby, The influence of microstructure on the fracture behavior of particulate metal matrix compositesMater. Sci. Engng. A135 (1991) 221-224.

4. T. Christman, A. Needleman, S. Nutt and S. Suresh, On microstructural evolution and micromechanical modelling of deformation of a whisker-reinforced metal-matrix composite. Mater. Sci. Eng., A107 (1989) 49-61.

5. R.M. German, A. Bose, Fabrication of intermetallic matrix composites. Mater. Sci. Eng., A107 (1989) 107-116.

6. Needleman, S.R. Nutt, S. Suresh, V. Tvergaard, Matrix, reinforcement and interfacial failure. In: S. Suresh, A. Mortensen and A. Needleman (eds.), Fundamentals of Metal Matrix Composites, Butterworth-Heinemann, Boston, MA., (1993) 233-250.

7. S.R. Nutt, A. Needleman, Void nucleation at fiber ends in Al-SiC composites. Scripta Metallurgica 21 (1987) 705-710.

8. V. Tvergaard, Effect of fibre debonding in a whisker-reinforced metal. Mater. Sci. Eng. A125 (1990) 203-213.

9. V. Tvergaard, Model studies of fibre breakage and debonding in a metal reinforced by short fibres. J. Mech. Phys. Solids 41 (1993) 1309-1326.

10. V. Tvergaard, Fibre debonding and breakage in a whisker-reinforced metal. Materials Science and Engineering A190 (1995) 215-222.

11. V. Tvergaard, Breakage and debonding of short brittle fibres among particulates in a metal matrix. Materials Science and Engineering A369 (2004) 192-200.

12. A. Levy, J.M. Papazian, Tensile properties of a short fiber-reinforced SiC/Al composites: Part II. Finite element analysis. Metall Trans. 21A (1990) 411-420.

13. Hom, C.L., Three-dimensional finite element analysis of plastic deformation in a whisker-reinforced metal matrix composite. J. Mech. Phys. Solids 40 (1992) 991-1008.

14. R.F. Bishop, R.F., R. Hill, N.F. Mott, The theory of indentation and hardness tests. Proc. Phys. Soc. 57 (1945) 147-159.

15. R. Hill, The mathematical theory of plasticity. Clarendon Press (1950) Oxford.

16. Y. Huang, J.W. Hutchinson, V. Tvergaard, Cavitation instabilities in elastic-plastic solids. J. Mech. Phys. Solids 39 (1989) 223-241.

17. V. Tvergaard, Y. Huang, J.W. Hutchinson, Cavitation instabilities in a power hardening elastic-plastic solid. Eur. J. Mech. A/Solids , 11 (1992) 215-231.

18. C.F. Niordson, V. Tvergaard, Size-effects on cavitation instabilities. Trans. ASME, E, J. Appl. Mech. 73 (2006) 246-253.

19. B.N. Legarth, V. Tvergaard, V., 3D analyses of cavitation instabilities accounting for plastic anisotropy. ZAMM 90 (2010) 701-709.

20. T.C. Kennedy, T. Puttapitukporn, M.E. Kassner, Dynamic effects on cavitation instabilities in solids. Acta Mechanica, 165 (2003) 73-85.

21. J.M. Ball, Discontinuous equilibrium solutions and cavitation in nonlinear elasticity. Phil. Trans. R. Soc. London, A306 (1982) 557-610. 
22. C.O. Horgan, R. Abeyaratne, A bifurcation problem for a compressible nonlinearly elastic medium: Growth of a microvoid, J. Elasticity , 16 (1986) 189-200.

23. C.O. Horgan, D.A. Polignone, Cavitation in nonlinearly elastic solids: A review. Appl. Mech. Rev., 48 (1995) 471-485.

24. M.F. Ashby, F.J. Blunt, M. Bannister, Flow characteristics of highly constrained metal wires. Acta Metall., 37 (1989) 1847-1857.

25. V. Tvergaard, Studies of void growth in a thin ductile layer between ceramics. Computational Mechanics 20 (1997) 186-191.

26. J.W. Hutchinson, Finite strain analysis of elastic-plastic solids and structures. In: Hartung, R.F. (Ed.), Numerical Solution of Nonlinear Structural Problems. ASME, New York (1973) 17.

27. V. Tvergaard, Effect of thickness inhomogeneities in internally pressurized elasticplastic spherical shells. J. Mech. Phys. Solids 24 (1976) 291-304.

28. V. Tvergaard, On cavitation instabilities with interacting voids. Eur. J. Mechanics A/Solids 32 (2012) 52-58. 
Figure captions:

Fig. 1. Periodic array of staggered fibres. (a) Cross-section along fibres. (b) Cross-section transverse to fibres.

Fig. 2. Axisymmetric unit cell containing part of a fibre and part of a void.

Fig. 3. Example of a mesh used for a unit cell containing void and fibre.

Fig. 4. Cavitation limit for a spherical void in an infinite elastic-plastic power hardening solid subject to remote axisymmetric stressing, for $\sigma_{Y} / E=0.005, v=0.3$ and $n=7.66$.

Fig. 5. Evolution of mean stress $\sigma_{m}$ at origo for four different fibre arrangements, when $\rho=0.5$ (there is no void). The reference case has $l_{c} / r_{c}=6, r_{f} / r_{c}=0.6$ and $l_{v} / r_{c}=0.8$. Deviations from the reference values are indicated on the curves.

Fig. 6. Evolution of mean stress $\sigma_{m}$ at origo for four different fibre arrangements, when $\rho=0$ (there is no void). The reference case has $l_{c} / r_{c}=6, r_{f} / r_{c}=0.6$ and $l_{v} / r_{c}=0.8$. Deviations from the reference values are indicated on the curves.

Fig. 7. Evolution of mean stress $\sigma_{m}$ at origo for four different fibre arrangements, when $\rho=0.25$ (there is no void). The reference case has $l_{c} / r_{c}=6, r_{f} / r_{c}=0.6$ and $l_{v} / r_{c}=0.8$. Deviations from the reference values are indicated on the curves.

Fig. 8. Evolution of the normalized void volume vs. axial strain for $l_{c} / r_{c}=6, r_{f} / r_{c}=0.6$, $l_{v} / r_{c}=0.8$ and $\rho=0.5$. The four different initial void radii are indicated on the curves.

Fig. 9. Deformed meshes for two different unit cells with $l_{c} / r_{c}=6, r_{f} / r_{c}=0.6, l_{v} / r_{c}=0.8$ and $\rho=0.5$. (a) For $r_{v} / r_{c}=0.01$ at $V / V_{0}=4108$. (b) For $r_{v} / r_{c}=0.1$ at $V / V_{0}=886$.

Fig. 10. Evolution of the normalized void volume vs. axial strain for four different fibre arrangements, when $\rho=0.5$ and $r_{v} / r_{c}=0.001$. The reference case has $l_{c} / r_{c}=6$, $r_{f} / r_{c}=0.6$ and $l_{v} / r_{c}=0.8$. Deviations from the reference values are indicated on the curves.

Fig. 11. Evolution of the normalized void volume vs. axial strain for four different fibre arrangements, when $\rho=0.0$ and $r_{v} / r_{c}=0.001$. The reference case has $l_{c} / r_{c}=6$, $r_{f} / r_{c}=0.6$ and $l_{v} / r_{c}=0.8$. Deviations from the reference values are indicated on the curves. 
Fig. 12. Evolution of the normalized void volume vs. axial strain for four different fibre arrangements, when $\rho=0.25$ and $r_{v} / r_{c}=0.001$. The reference case has $l_{c} / r_{c}=6$, $r_{f} / r_{c}=0.6$ and $l_{v} / r_{c}=0.8$. Deviations from the reference values are indicated on the curves.
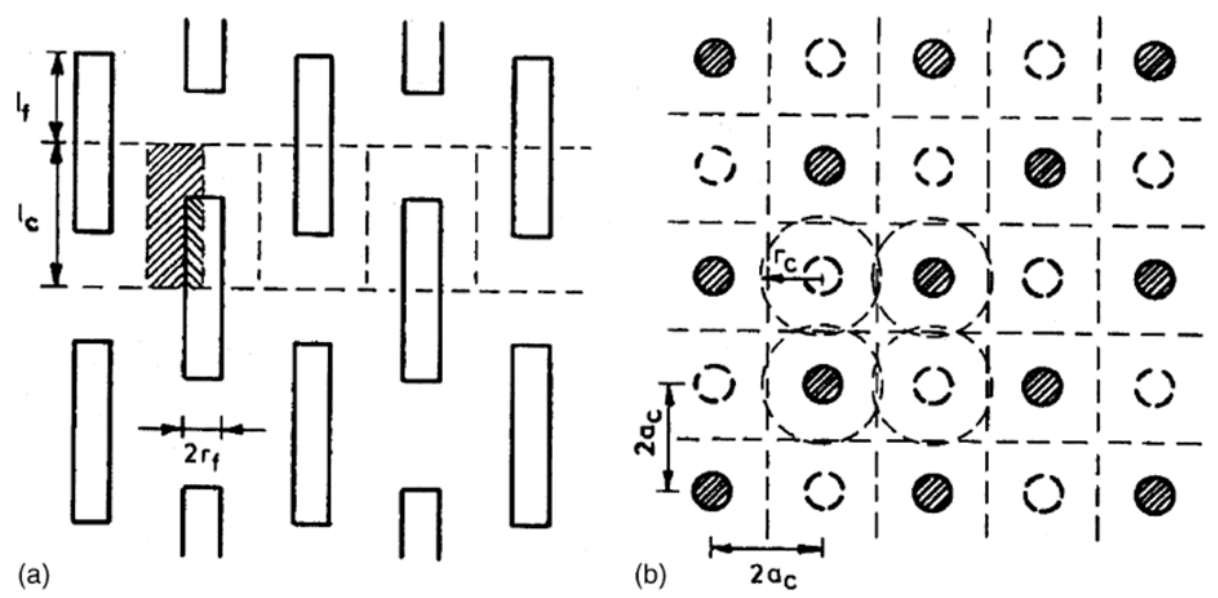

Fig. 1. Periodic array of staggered fibres. (a) Cross-section along fibres. (b) Cross-section transverse to fibres.

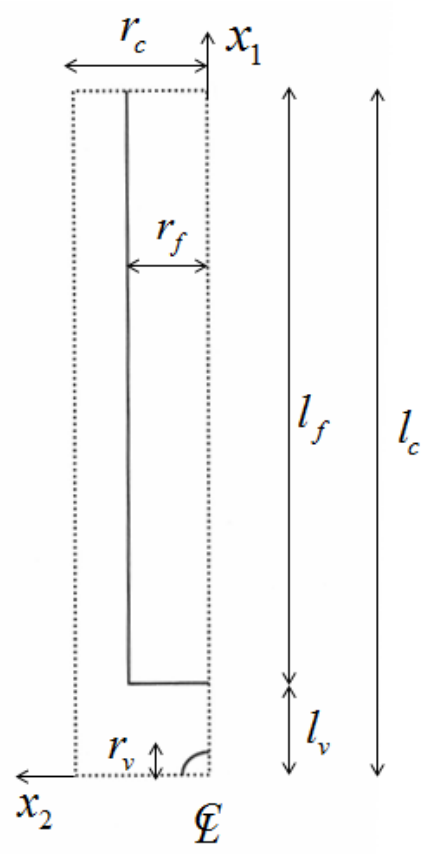


Fig. 2. Axisymmetric unit cell containing part of a fibre and part of a void.

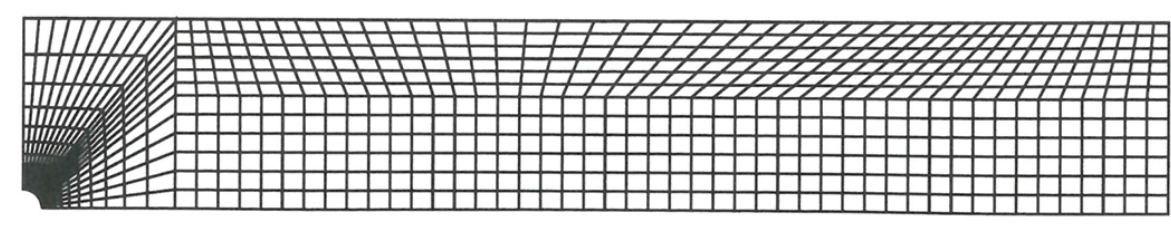

Fig. 3. Example of a mesh used for a unit cell containing void and fibre.

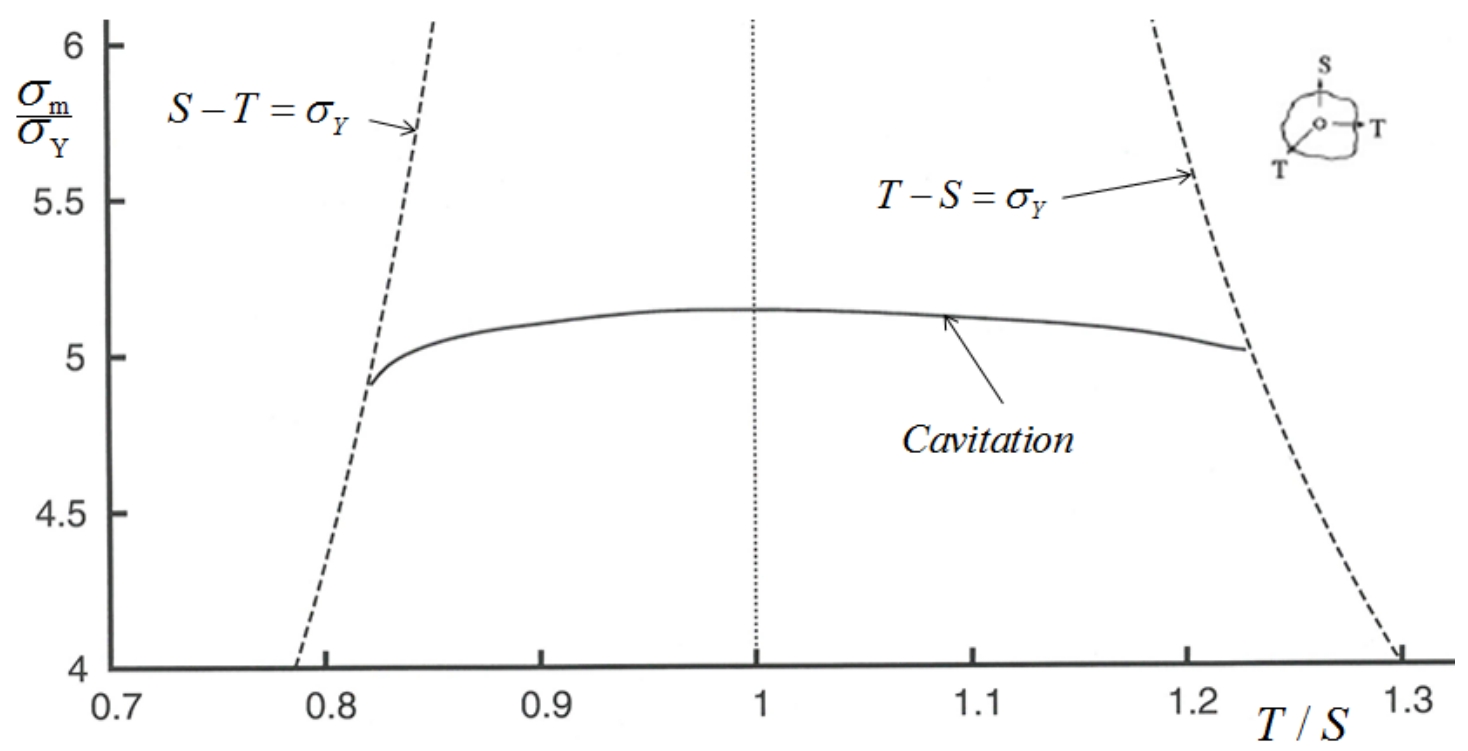

Fig. 4. Cavitation limit for a spherical void in an infinite elastic-plastic power hardening solid subject to remote axisymmetric stressing, for $\sigma_{Y} / E=0.005, v=0.3$ and $n=7.66$. 


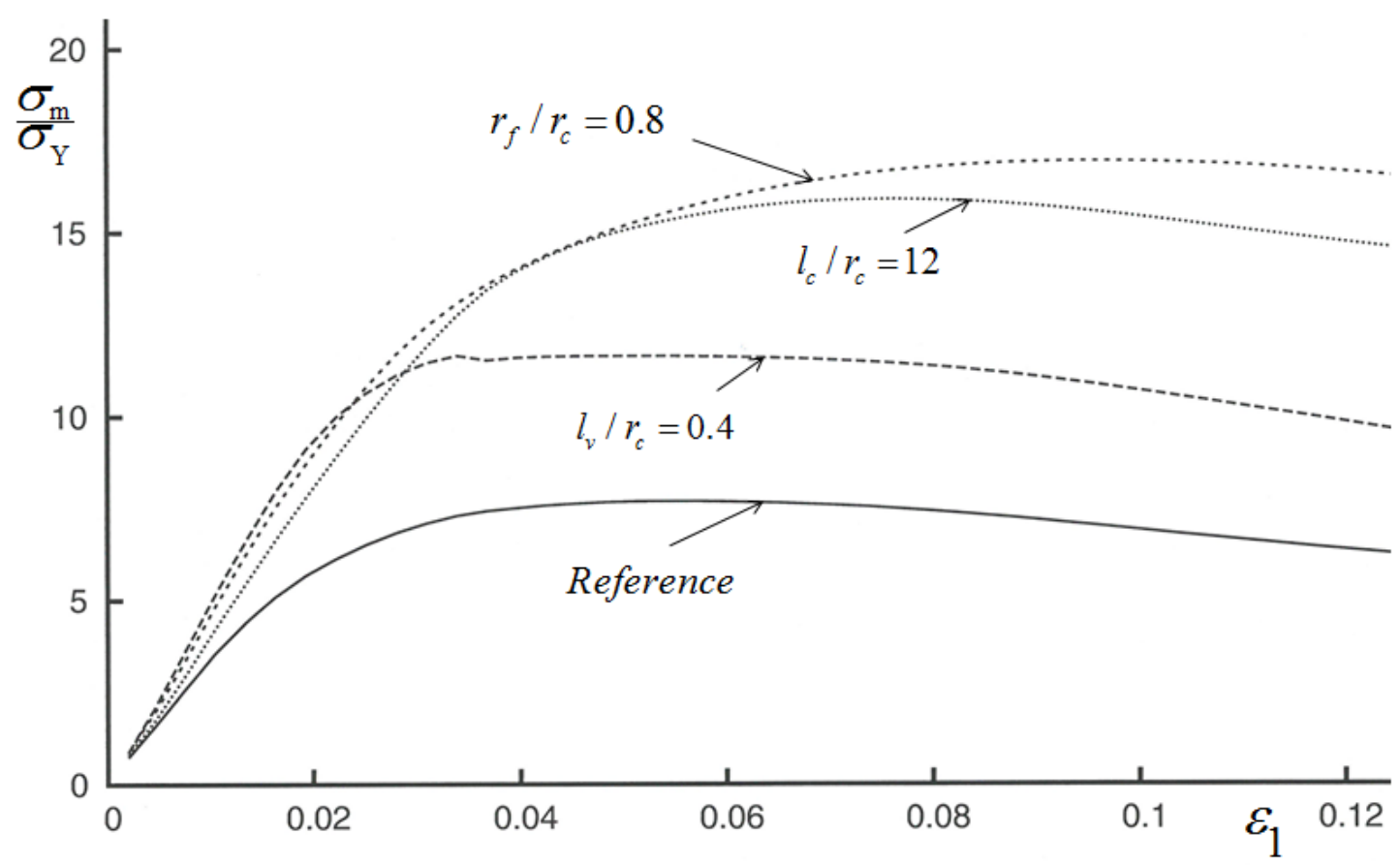

Fig. 5. Evolution of mean stress $\sigma_{m}$ at origo for four different fibre arrangements, when $\rho=0.5$ (there is no void). The reference case has $l_{c} / r_{c}=6, r_{f} / r_{c}=0.6$ and $l_{v} / r_{c}=0.8$. Deviations from the reference values are indicated on the curves.

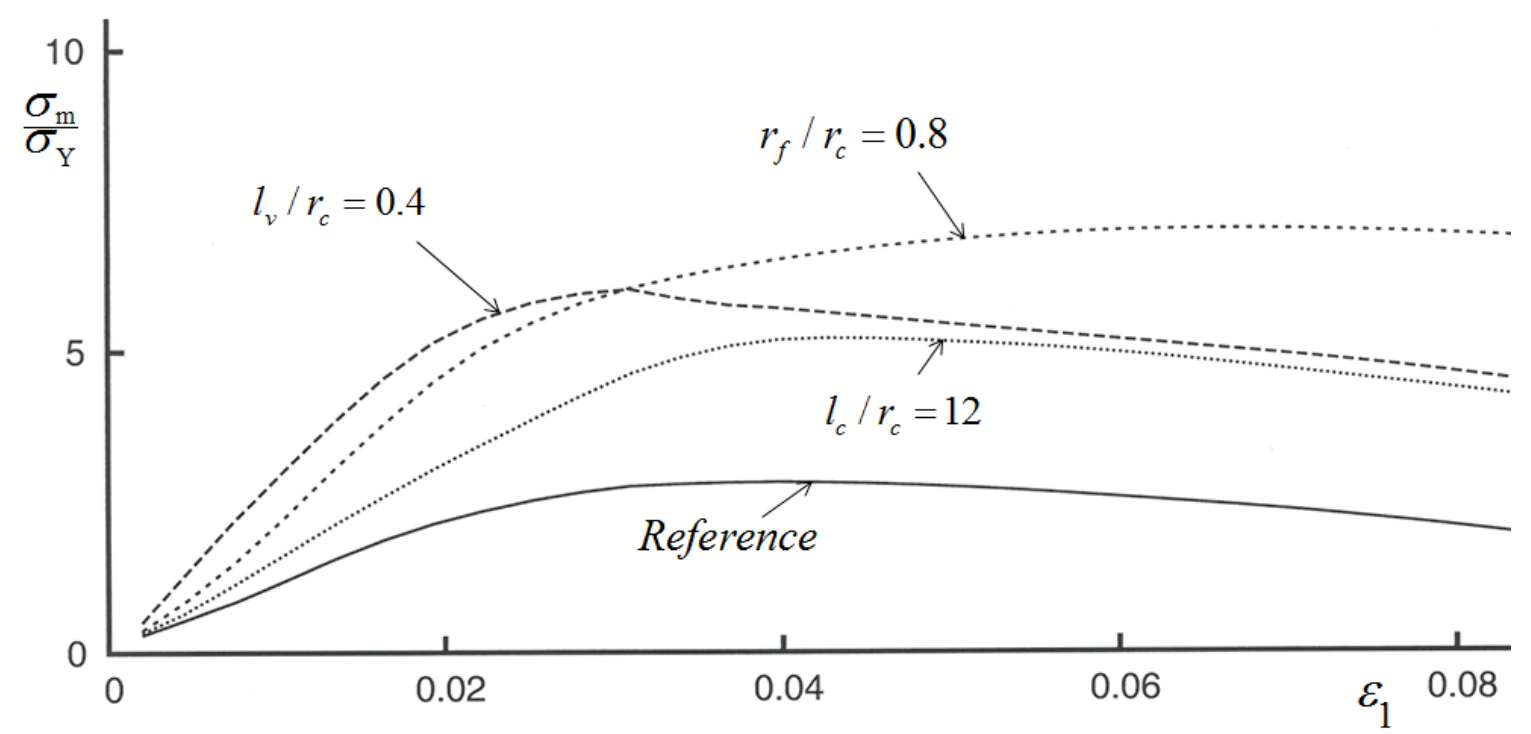

Fig. 6. Evolution of mean stress $\sigma_{m}$ at origo for four different fibre arrangements, when $\rho=0$ (there is no void). The reference case has $l_{c} / r_{c}=6, r_{f} / r_{c}=0.6$ and $l_{v} / r_{c}=0.8$. Deviations from the reference values are indicated on the curves. 


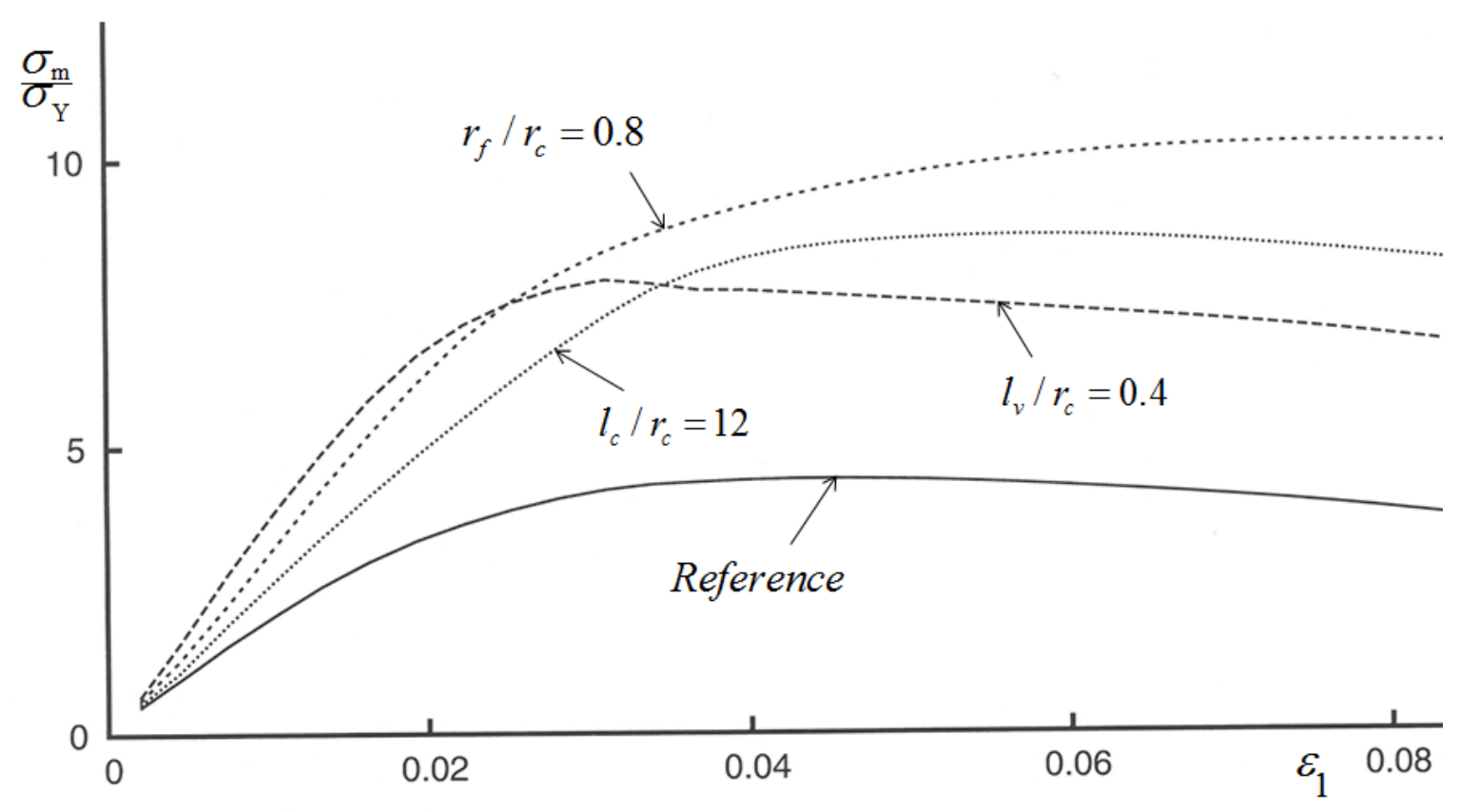

Fig. 7. Evolution of mean stress $\sigma_{m}$ at origo for four different fibre arrangements, when $\rho=0.25$ (there is no void). The reference case has $l_{c} / r_{c}=6, r_{f} / r_{c}=0.6$ and $l_{v} / r_{c}=0.8$. Deviations from the reference values are indicated on the curves.

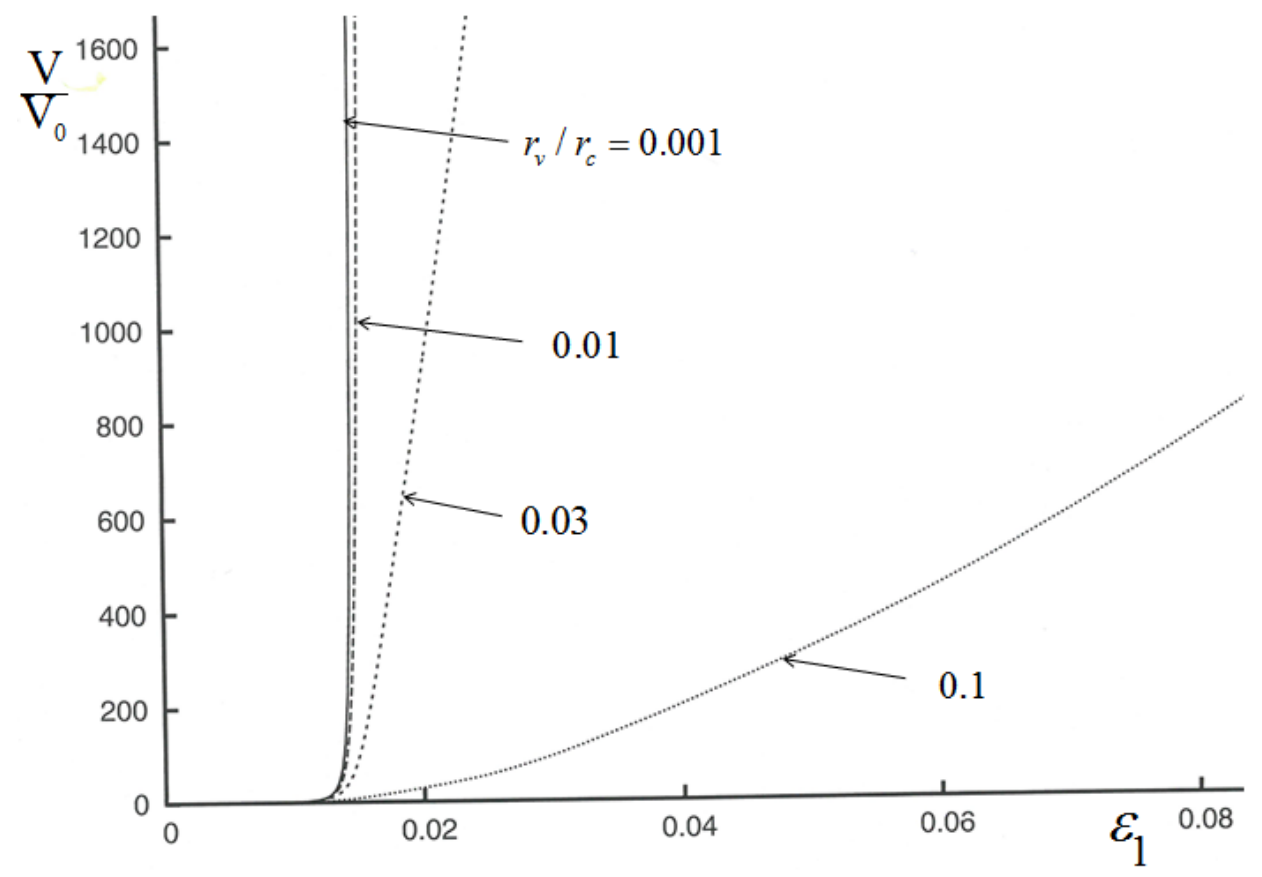

Fig. 8. Evolution of the normalized void volume vs. axial strain for $l_{c} / r_{c}=6, r_{f} / r_{c}=0.6$, $l_{v} / r_{c}=0.8$ and $\rho=0.5$. The four different initial void radii are indicated on the curves. 


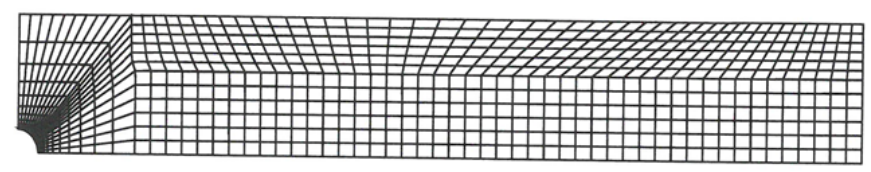

(a)



(b)

Fig. 9. Deformed meshes for two different unit cells with $l_{c} / r_{c}=6, r_{f} / r_{c}=0.6, l_{v} / r_{c}=0.8$ and $\rho=0.5$. (a) For $r_{v} / r_{c}=0.01$ at $V / V_{0}=4108$. (b) For $r_{v} / r_{c}=0.1$ at $V / V_{0}=886$.



Fig. 10. Evolution of the normalized void volume vs. axial strain for four different fibre arrangements, when $\rho=0.5$ and $r_{v} / r_{c}=0.001$. The reference case has $l_{c} / r_{c}=6$, $r_{f} / r_{c}=0.6$ and $l_{v} / r_{c}=0.8$. Deviations from the reference values are indicated on the curves. 


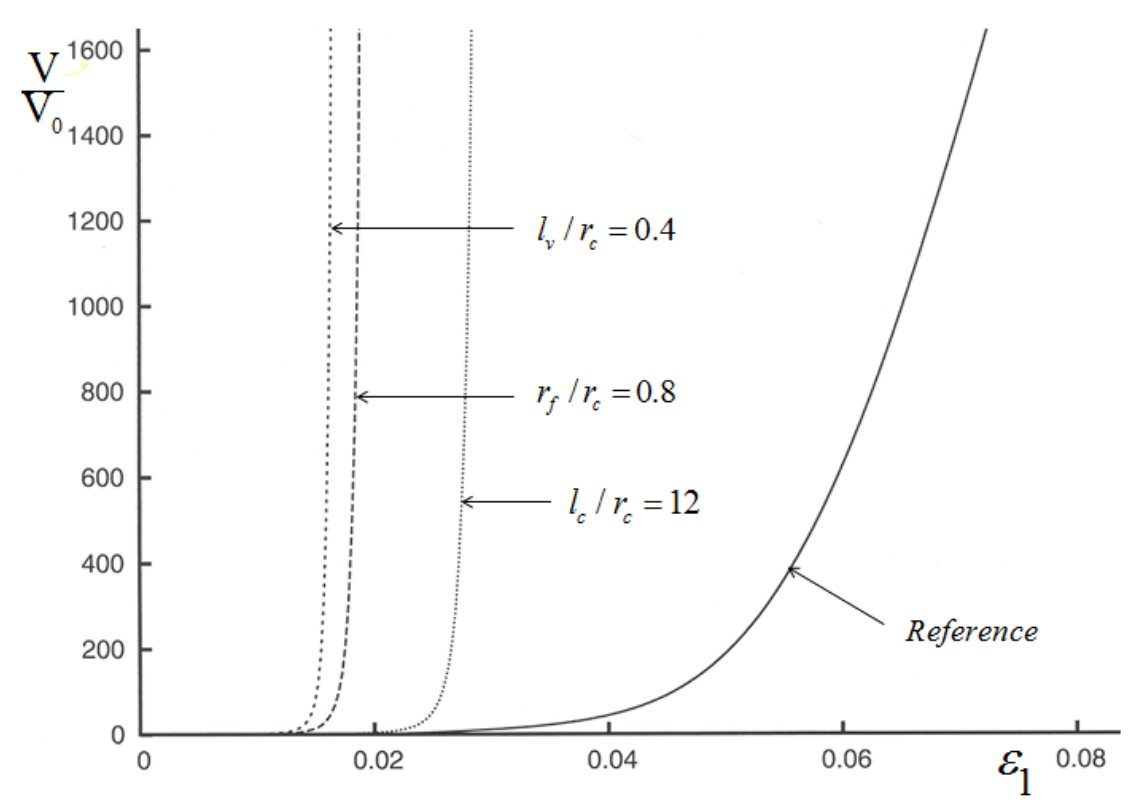

Fig. 11. Evolution of the normalized void volume vs. axial strain for four different fibre arrangements, when $\rho=0.0$ and $r_{v} / r_{c}=0.001$. The reference case has $l_{c} / r_{c}=6$, $r_{f} / r_{c}=0.6$ and $l_{v} / r_{c}=0.8$. Deviations from the reference values are indicated on the curves.

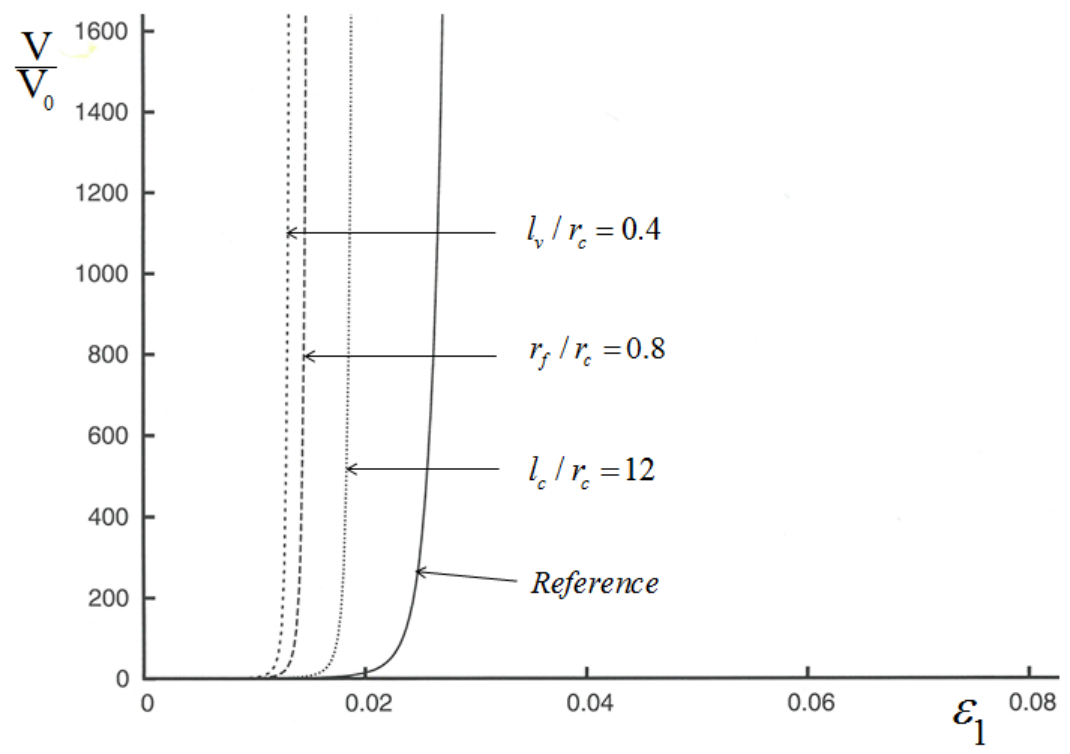

Fig. 12. Evolution of the normalized void volume vs. axial strain for four different fibre arrangements, when $\rho=0.25$ and $r_{v} / r_{c}=0.001$. The reference case has $l_{c} / r_{c}=6$, $r_{f} / r_{c}=0.6$ and $l_{v} / r_{c}=0.8$. Deviations from the reference values are indicated on the curves. 
